\title{
LIMIT OF THE TRANSPORT CAPACITY OF A DENSE WIRELESS NETWORK
}

\author{
RADHA KRISHNA GANTI* AND \\ MARTIN HAENGGI,** University of Notre Dame
}

\begin{abstract}
It is known that the transport capacity of a dense wireless ad hoc network with $n$ nodes scales like $\sqrt{n}$. We show that the transport capacity divided by $\sqrt{n}$ approaches a nonrandom limit with probability 1 when the nodes are uniformly distributed on the unit square. To show the existence of the limit, we prove that the transport capacity under the protocol model is a subadditive Euclidean functional and use the machinery of subadditive functions in the spirit of Steele.
\end{abstract}

Keywords: Subadditive Euclidean functional; transport capacity; ad hoc network

2010 Mathematics Subject Classification: Primary 60D05

Secondary 46N30; 47N30

\section{Introduction}

Consider a wireless network of $n$ radio transceivers (nodes) $X_{n}=\left\{x_{1}, \ldots, x_{n}\right\}$ distributed uniformly and independently on the unit square ( $x_{i}$ is used to identify both the node and its location). Since the medium is wireless, the signal transmitted by any node effectively spreads throughout the complete space and interferes with other concurrent transmissions. We use the protocol model introduced by Gupta and Kumar [3] to model the connectivity between a source and destination in the presence of interference.

Protocol model. A node $x_{i}$ can transmit information to a node $x_{j}$ if and only if the closed disk centered around the receiver $x_{j}$ with radius $\beta\left\|x_{i}-x_{j}\right\|, \beta>1$, has no other transmitter. This is to make sure that there are no interferers close to the receiver. If the set of transmitters is denoted by $S$ and the receivers by $R$, and $S \cap R=\varnothing$, then, by the protocol model, if

$$
B\left(x_{j}, \beta\left\|x_{i}-x_{j}\right\|\right) \cap S=\left\{x_{i}\right\}
$$

then the transmitter $x_{i}$ can communicate with the receiver $x_{j}$. Here $B(x, r)$ denotes a closed disc of radius $r$ centered at $x$. Also, we allow each transmitter to communicate with only one receiver, i.e. broadcasting is not permitted. A node can either transmit or receive at a given instant but not both.

We can immediately observe that one can choose transmitter-receiver pairs in numerous ways such that (1) holds for all these pairs. So a metric called the transport capacity (TC) was introduced in [3] and the transmitter-receiver pairs are chosen so as to maximize the TC. The

Received 8 December 2008; revision received 9 June 2010.

* Current address: Department of Electrical Engineering, University of Texas at Austin, Austin TX, 78712, USA.

Email address: rganti@austin.utexas.edu

** Current address: Department of Electrical Engineering, University of Notre Dame, Notre Dame, IN 46556, USA.

Email address: mhaenggi@nd.edu 
TC of the node set $X_{n}$ using single hop is defined as

$$
\mathrm{T}\left(X_{n}\right)=\max _{\substack{r \in \mathcal{R}_{S} \\ S \subseteq X_{n}}} \sum_{x \in S}\|x-r(x)\| \mathbf{1}(x \rightarrow r(x), S),
$$

where $\mathcal{R}_{S}$ is the set of all injective functions from $S$ to $R$. Here $\mathbf{1}(x \rightarrow r(x), S)$ is equal to 1 if (1) is satisfied for the transmitter $x$ and receiver $r(x)$ when the transmitting set is $S$, and 0 otherwise. We showed in [2] that (2) is equivalent to the multi-hop transport capacity where information can be transmitted using multiple hops between a source and destination. While it has been proved that the TC scales like $\sqrt{n}$ [1], [3], the question of whether the limit $\lim _{n \rightarrow \infty} \mathrm{T}\left(X_{n}\right) / \sqrt{n}$ exists remains open. In this paper we prove the following.

Theorem 1. Let $X_{n}=\left\{x_{1}, x_{2}, \ldots, x_{n}\right\}$ denote $n$ nodes independently uniformly distributed in $[0,1]^{2}$. For the transport capacity, we have

$$
\lim _{n \rightarrow \infty} \frac{\mathrm{T}\left(X_{n}\right)}{\sqrt{n}}=\mathrm{D}_{2}
$$

with probability 1 and

$$
\mathrm{P}\left(\left|\mathrm{T}\left(X_{n}\right)-\mathrm{ET}\left(X_{n}\right)\right| \geq t\right) \leq C \exp \left(-C_{1} \frac{t^{4}}{n}\right),
$$

where $\mathrm{D}_{2}, C$, and $C_{1}$ are positive constants that do not depend on $n$.

The technique in this paper can be easily extended to show that

$$
\lim _{n \rightarrow \infty} \mathrm{T}\left(X_{n}\right) n^{-(d-1) / d}=\mathrm{D}_{d} \quad \text { almost surely }
$$

when the nodes $x_{i}$ are independently uniformly distributed in $[0,1]^{2}$, and $\mathrm{D}_{d}$ is a constant depending only on $\beta$ and the dimension $d$.

\section{Proof}

We prove Theorem 1 by showing that $\mathrm{T}$ is a subadditive Euclidean functional and, hence, the limit in (3) follows from the results of Steele [6] and Rhee [5], which we state here for convenience.

Theorem 2. ([6].) Suppose that $\mathrm{T}$ is a monotone, subadditive Euclidean functional on $\mathbb{R}^{d}$ with finite variance. Then there is a constant $\delta(\mathrm{T})$ such that

$$
\lim _{n \rightarrow \infty} \frac{\mathrm{T}\left(X_{n}\right)}{n^{(d-1) / d}}=\delta(\mathrm{T}) .
$$

Theorem 3. ([5].) If, in addition to the conditions in Theorem 2, for any finite subsets $F$ and $G$ of $[0,1]^{d}$, there exists a constant $C_{2}$ such that

$$
\mathrm{T}(F \cup G)-\mathrm{T}(F) \leq C_{2}|G|^{(d-1) / d},
$$

where $|G|$ is the cardinality of the set $G$, then, for some constants $C_{3}$ and $C_{4}$,

$$
\mathrm{P}\left(\left|\mathrm{T}\left(X_{n}\right)-\mathrm{ET}\left(X_{n}\right)\right| \geq t\right) \leq C_{3} \exp \left(-\frac{C_{4} t^{2 d /(d-1)}}{n}\right) .
$$


We will use $(x \rightarrow y)$ to denote the transmission with $x$ as the transmitter and $y$ as the receiver.

Lemma 1. For a transmitter receiver pair $(x, y)$, define

$$
D(x, y)=\bigcup_{z \in \operatorname{line}(x, y)} B\left(z, \frac{\beta-1}{2}\|x-y\|\right),
$$

i.e. the $(\beta-1)\|x-y\| / 2$ neighborhood of the line joining $x$ and $y$. If $\left(x_{1} \rightarrow y_{1}\right)$ and $\left(x_{2} \rightarrow y_{2}\right)$ are two pairs of successful transmissions, then $D\left(x_{1}, y_{1}\right) \cap D\left(x_{2}, y_{2}\right)=\varnothing$.

Proof. The proof of the above is identical to the proof of Theorem 3.3 of [4].

Lemma 1 indicates that each successful transmission occupies a certain area of the Euclidean plane which is disjoint with the corresponding region of other successful transmissions. Let $\left(x_{i} \rightarrow y_{i}\right), 1 \leq i \leq m$, be a set of transmissions in a square of area $A$. So from Lemma 1 we have the following necessary condition for all the transmissions to be successful:

$$
\frac{\pi}{4} \frac{(\beta-1)^{2}}{4} \sum_{i=1}^{m}\left\|x_{i}-y_{i}\right\|^{2}<A .
$$

For the above necessary condition, we use the fact that a ball of radius $(\beta-1)\left\|x_{i}-y_{i}\right\| / 2$ around each receiver (for a successful transmission) should be disjoint from each other. The factor $\pi / 4$ arises because a receiver may be located on the vertex of the square $A$. Using (5) and the Cauchy-Schwarz inequality, it was first shown in [4] that the transport capacity of $n$ nodes in a square of area $A$ is upper bounded by

$$
\mathrm{T}\left(X_{n}\right)<c \sqrt{A n} .
$$

Lemma 2. Consider a square $A=[0, t]^{2}$. Consider the scenario in which transmitters in A can only communicate with receivers outside the square A. If we restrict the maximum transmitter-receiver distance to be $c_{1} t$ (to prevent the case where a single transmitter in A can communicate with a receiver at an arbitrary distance located outside A), then the transport capacity in this setup is upper bounded by $c_{2} t$.

Proof. See Figure 1. For all the successful transmitter-receiver pairs $(x, y)$, the regions $D(x, y)$ are disjoint. In our case the transmitters are inside the square $[0, t]^{2}$. Let the contending transmitter receiver distances be $\left\{r_{1}, r_{2}, \ldots, r_{n}\right\}$. Since the receivers are outside the box and each transmission cuts the boundary, we have

$$
2 \frac{\beta-1}{2}\left(r_{1}+r_{2}+\cdots+r_{n}\right) \leq 4 t .
$$

Hence, the transport capacity in this case is upper bounded by $\max \left\{4 t /(\beta-1), c_{1} t\right\}$.

Lemma 3. Consider a square $A=[0, t]^{2}$, and let $X_{n}=\left\{x_{1}, \ldots, x_{n}\right\} \subset A$ denote a set of $n$ nodes. Divide A into $m^{2}$ squares of equal sides with length $t / m$, and denote the subsquares by $A_{1}, \ldots, A_{m^{2}}$. Then

$$
\mathrm{T}\left(X_{n}\right) \leq \sum_{i=1}^{m^{2}} \mathrm{~T}\left(X_{n} \cap A_{i}\right)+C m t
$$




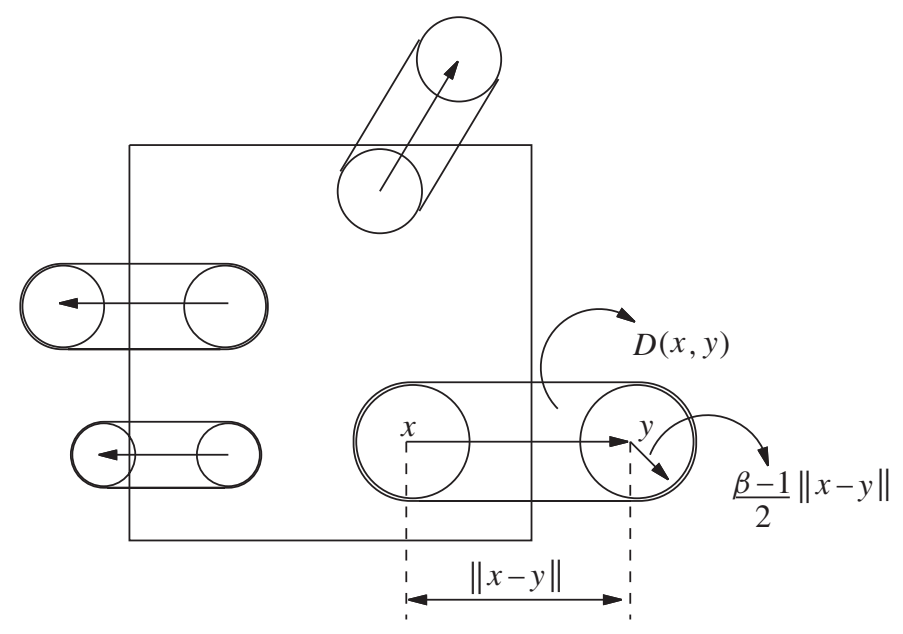

Figure 1: Illustration of the proof of Lemma 2. The sausage regions represent $D(x, y)$ for different transmitter-receiver pairs.

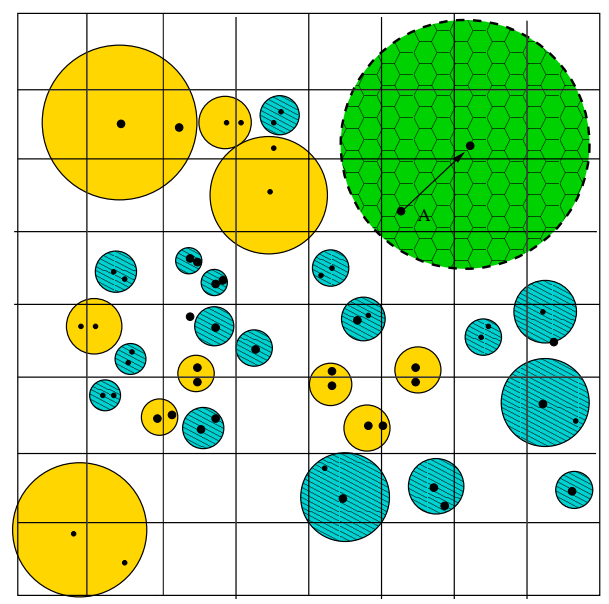

Figure 2: Proof technique for Lemma 3. The dark hashed circles correspond to $\left(A_{i} \rightarrow A_{i}\right)$ and the TC contribution can be bounded by $\mathrm{T}\left(A_{i}\right)$. The unhashed circles correspond to $\left(A_{i} \rightarrow A_{i}^{\mathrm{c}}\right)$. However, these cannot contribute much to the TC by Lemma 2. The maximum contribution from these is $\mathrm{cm}^{2} t / \mathrm{m}=\mathrm{cmt}$. Observe that when the transmitter-receiver distance is greater than $a=2 \sqrt{2} t /(m(\beta-1))$, there can be a maximum of one transmission per square (as in the top-right comb circle).

Proof. We focus on a single square $A_{i}$. There are three types of transmissions, $\left(A_{i} \rightarrow A_{i}\right)$, $\left(A_{i} \rightarrow A_{i}^{\mathrm{c}}\right)$, and $\left(A_{i}^{\mathrm{c}} \rightarrow A_{i}\right)$. See Figure 2. The contribution of transmissions from $A_{i}$ into $A_{i}$ towards the total distance that the information is transmitted can be upper bounded by $\mathrm{T}\left(X_{n} \cap A_{i}\right)$. Hence, the total contribution by $\left(A_{i} \rightarrow A_{i}\right), 1 \leq i \leq m^{2}$, is upper bounded by $\sum_{i=1}^{m^{2}} \mathrm{~T}\left(X_{n} \cap A_{i}\right)$. The only transmissions which involve $A_{i}$ to be accounted for are $\left(A_{i} \rightarrow A_{i}^{\mathrm{c}}\right)$ and $\left(A_{i}^{\mathrm{c}} \rightarrow A_{i}\right)$. The contribution of these transmissions towards the total distance that information propagates is denoted by $\tilde{T}$, and $\tilde{T}$ upper bounds the TC. Let $F\left(A_{k}\right)$ denote a set of feasible transmitters in square $A_{k}$ with receivers in $A_{k}^{\mathrm{c}}$. By the sphere-packing bound (5) 
we have

$$
\sum_{k=1}^{m^{2}} \sum_{x \in F\left(A_{k}\right)}\|x-r(x)\|^{2} \leq C t^{2} .
$$

Let $b_{k}=\sum_{x \in F\left(A_{k}\right)}\|x-r(x)\|$. So we require to bound $\tilde{T}=\sup \left\{\sum_{k=1}^{m^{2}} b_{k}\right\}$, where the supremum is taken over all the feasible sets of transmissions. Let the number of squares with all of their transmission distances less than $a=2 \sqrt{2} t /(m(\beta-1)$ ) be $\eta$ (so in each of these squares, every transmitter chooses a receiver outside the square at a distance less than $a$ ). Denote this set of squares by $C_{a} \subset\left\{1, \ldots, m^{2}\right\}$. So we have $\left|C_{a}\right|=\eta$ and $\tilde{T}=\sup \left\{\sum_{k \in C_{a}} b_{k}+\sum_{k \in C_{a}^{\mathrm{c}}} b_{k}\right\}$, where $|\cdot|$ denotes the cardinality of the set. Let $A_{k} \in C_{a}^{\mathrm{c}}$. We then have $\left|F\left(A_{k}\right)\right|=1$. Since the maximum number of transmitters is $m^{2}-\eta$, we have

$$
\sum_{k \in C_{a}^{\mathrm{c}}} b_{k} \leq c_{1} t \sqrt{m^{2}-\eta} .
$$

For the other set $C_{a}$ with transmitter-receiver distances less than $a$, by Lemma 2, the transport capacity is upper bounded by

$$
\sum_{k \in C_{a}} b_{k} \leq c_{2} \frac{t}{m} \eta
$$

So we have

$$
\tilde{T} \leq c_{1} t \sqrt{m^{2}-\eta}+c_{2} \frac{t}{m} \eta, \quad 0 \leq \eta \leq m^{2} .
$$

The maximum value of the right-hand side for the given range of $\eta$ is $\mathrm{ctm}$, which concludes the proof.

Proof of Theorem 1. We have the following conditions.

(A1) $\mathrm{T}\left(a X_{n}\right)=a \mathrm{~T}\left(X_{n}\right)$ for all $a>0$. This follows from the definition.

(A2) $\mathrm{T}\left(X_{n}+x\right)=\mathrm{T}\left(X_{n}\right)$ for all $x \in \mathbb{R}^{2}$, where $X_{n}+x=\left\{x_{1}+x, x_{2}+x, \ldots, x_{n}+x\right\}$. This follows from the definition.

(A3) $\mathrm{T}\left(X_{n} \cup\{x\}\right) \geq \mathrm{T}\left(X_{n}\right)$, since the supremum in (2) is taken over a bigger set (we may just neglect the newly added point).

(A4) Finite variance: $\operatorname{var} \mathrm{T}\left(X_{n}\right)<\infty$, follows from (6).

(A5) For $F, G \subset[0,1]^{2}$ and $F, G$ finite (observe that $F$ and $G$ need not be disjoint), we have

$$
\mathrm{T}(F \cup G)-\mathrm{T}(G)<c \sqrt{|F|},
$$

where $c$ is a constant that does not depend on $F$ and $G$. The transmissions can be partitioned into $(G \rightarrow G),(F \rightarrow F),(G \rightarrow F)$, and $(F \rightarrow G)$. The contribution of the transmission $(G \rightarrow G)$ to TC can be upper bounded by $\mathrm{T}(G)$. Observe that the maximum cardinality of the remaining transmissions can be $|F|$. So we have

$$
\mathrm{T}(F \cup G)<\mathrm{T}(G)+c \sqrt{|F|} .
$$

Conditions (A1), (A2), (A3), and (A4) indicate that $\mathrm{T}$ is a monotone, subadditive Euclidean functional with finite variance. Hence, (3) follows from Theorem 2, and (4) follows from Theorem 3 and (A5). 
Lemma 4. The limit $\mathrm{D}_{2}$ in Theorem 1 is positive (i.e. $\mathrm{D}_{2}>0$ ) when the nodes are uniformly i.i.d. on the unit square.

Proof. We will prove that $\mathrm{E} \mathrm{T}\left(X_{n}\right)>C \sqrt{n}$ for some $C>0$. Then $\mathrm{D}_{2}>0$ follows from (4). Consider a tessellation of the unit square by subsquares of side length $1 / \sqrt{n}$. Each subsquare is denoted by $s_{i, j}$, where $i$ represents the row number and $j$ represents the column number. Each subsquare might have a point (even more than one) or none. Let the transmitter set be

$$
\begin{array}{r}
S=\left\{x: x \in X_{n} \cap s_{k \delta, l \delta} \text { and }\|x-(k \delta, l \delta)\| \leq\|y-(k \delta, l \delta)\|\right. \\
\left.\quad \text { for all } y \in X_{n} \cap s_{k \delta, l \delta}, k, l \in \mathbb{Z}^{+}\right\},
\end{array}
$$

where $\delta=\lceil\sqrt{10} \beta+4\rceil$ (alternatively, $S$ can formed by choosing a node at random from each subsquare). For each transmitter, choose a node (if it exists) in the second right adjacent cell as its receiver. So all the transmitter-receiver pairs are successful by our choice of transmitterreceiver pairs. So we have

$$
\mathrm{T}\left(X_{n}\right)>\frac{1}{\sqrt{n}} \sum_{S} \mathbf{1}\left(s_{k \delta, l \delta} \text { is not empty }\right) \mathbf{1}\left(s_{k \delta+2, l \delta}\right. \text { is not empty). }
$$

Taking the expectation we have

$$
\begin{aligned}
\mathrm{E} \mathrm{T}\left(X_{n}\right) & >\frac{\sqrt{n}}{\delta^{2}} \mathrm{P}\left(s_{0,0} \text { is not empty, } s_{2,0} \text { is not empty }\right) \\
& >\frac{\sqrt{n}}{\delta^{2}}\left[1-\mathrm{P}\left(s_{0,0} \text { is empty }\right)-\mathrm{P}\left(s_{2,0} \text { is empty }\right)\right] \\
& =\frac{\sqrt{n}}{\delta^{2}}\left[1-2\left(1-\frac{1}{n}\right)^{n}\right]
\end{aligned}
$$

So we have

$$
\mathrm{D}_{2}=\lim _{n \rightarrow \infty} \frac{\mathrm{ET}\left(X_{n}\right)}{\sqrt{n}}>\frac{1-2 \mathrm{e}^{-1}}{\delta^{2}}>0 .
$$

\section{Conclusion}

In this paper we have shown that the transport capacity of $n$ nodes distributed uniformly on a unit square, when normalized by $\sqrt{n}$, approaches a nonrandom limit. We have used the theory of subadditive Euclidean functionals to show the existence of the limit and a concentration inequality of the TC around its mean.

\section{Acknowledgements}

The support of the NSF (grants CNS 04-47869 and CCF 728763) and the DARPA/IPTO IT-MANET program (grant W911NF-07-1-0028) are gratefully acknowledged.

\section{References}

[1] Franceschetti, M., Dousse, O., Tse, D. N. C. and Thiran, P. (2007). Closing the gap in the capacity of wireless networks via percolation theory. IEEE Trans. Inf. Theory 53, 1009-1018.

[2] Ganti, R. K. And HaengGi, M. (2008). The transport capacity of a wireless network is a subadditive Euclidean functional. In Proc. IEEE Trans. Conf. Mobile Ad Hoc and Sensor Systems (Atlanta, GA), pp. 784-789. 
[3] Gupta, P. and Kumar, P. R. (2000). The capacity of wireless networks. IEEE Trans. Inf. Theory 46, $388-404$.

[4] Kumar, P. R. AND XUe, F. (2006). Scaling laws for ad hoc wireless networks: an information theoretic approach. In Foundations and Trends in Networking, Now Publishers, Hanover, MA, pp. 145-270.

[5] Rhee, W. T. (1993). A matching problem and subadditive Euclidean functionals. Ann. Appl. Prob. 3, $794-801$.

[6] Steele, J. M. (1981). Subadditive Euclidean functionals and nonlinear growth in geometric probability. Ann. Prob. 9, 365-376. 